\title{
Belphégor
}

\section{Desormeaux, Daniel. Alexandre Dumas, fabrique d'immortalité}

\section{Vittorio Frigerio}

\section{Q OpenEdition}

1 Journals

\section{Electronic version}

URL: http://journals.openedition.org/belphegor/718

DOI: 10.4000/belphegor.718

ISSN: 1499-7185

Publisher

LPCM

\section{Electronic reference}

Vittorio Frigerio, « Desormeaux, Daniel. Alexandre Dumas, fabrique d'immortalité », Belphégor [Online], 14 | 2016, Online since 16 July 2016, connection on 24 September 2020. URL : http:// journals.openedition.org/belphegor/718; DOI : https://doi.org/10.4000/belphegor.718

This text was automatically generated on 24 September 2020.

\section{(c) (i) (9)}

Belphégor est mis à disposition selon les termes de la Licence Creative Commons Attribution - Pas d'Utilisation Commerciale - Pas de Modification 4.0 International. 


\section{Desormeaux, Daniel. Alexandre Dumas, fabrique d'immortalité}

\section{Vittorio Frigerio}

\section{REFERENCES}

Desormeaux, Daniel. Alexandre Dumas, fabrique d'immortalité. Paris : Classiques Garnier. "Études romantiques et dix-neuviémistes », 43, 2014. 343 p.

Daniel Desormeaux

Alexandre Dumas,

fabrique d'immortalité 
1 Dumas est censé être devenu immortel, pour la culture institutionnelle du moins, depuis que ses restes ont été transférés au Panthéon. Drôle d'immortalité, on le sait, dictée tout autant par des considérations très contemporaines et politiques que par une quelconque remise en cause du jugement critique sur la valeur de l'œuvre de l'écrivain. Le livre de Desormeaux met la question de l'immortalité au centre de sa réflexion. Mais il y a une autre question qui vient spontanément à l'esprit du lecteur dès le titre. De quel Dumas parle-t-on? Il serait risqué de croire qu'il s'agit uniquement, ni même parfois surtout, du père. Il sera souvent question dans cet ouvrage tout autant du grand-père que du fils, et même parfois de la fille, Marie, romancière elle aussi quoiqu'on ait tendance à l'oublier. Et au-delà d'eux, de la permanence des écrivains dans la mémoire nationale et de la labilité de la littérature dans la mémoire humaine.

2 La conclusion de l'ouvrage ( Je finis en disant qu'Alexandre Dumas n'est qu'un encombrant héritier de Nodier » [p. 319]), dans sa brusquerie qui vient en quelque sorte contredire une lecture souvent fort complexe, nourrie d'une connaissance approfondie de la littérature et des littérateurs de l'époque, ne brille pas par sa subtilité. Encore faut-il voir comment le critique y est parvenu. Le parcours est en effet toujours intéressant, quoique parfois légèrement frustrant. L'ouvrage est organisé en trois parties. La première, qui comporte quatre sections, se concentre sur les rapports entre Dumas et Nodier et sur Les mille et un fantômes. La deuxième, qui ne comporte qu'une seule section, traite des Mémoires. La troisième, qui en comporte trois, passe des Mémoires au Dictionnaire de cuisine. Une introduction et une conclusion viennent comme il se doit montrer la voie de l'analyse et en tirer les fils au bout du parcours. Chaque section est axée autour d'un concept simple, qu'on peut souvent résumer en un seul mot (dérive, oubli, mémoire(s), immor(t)alité...). Cela présente des avantages et des désavantages. Parmi les premiers, le plus évident est l'identification d'un fil rouge que l'on suit à travers les œuvres évoquées. Parmi les deuxièmes, plus difficile à éviter, la pulsion à vouloir à tout prix faire rentrer dans le moule choisi la matière analysée, que celle-ci s'y prête immédiatement ou non. Ou alors la tendance à vouloir choisir sa matière en fonction de son intuition, mais il s'agit là d'un travers auquel nul critique n'échappe au fond et qu'on saurait par conséquent reprocher difficilement à l'auteur de cet ouvrage.

3 La thèse de l'étude est en définitive assez simple, et certainement assez clairement réitérée dans les trois cent et quelques pages de l'ouvrage : Dumas est à « la recherche désespérée d'une postérité » (p. 152). Il est aussi, on le sait après les travaux de Toesca et d'autres, à la recherche d'une origine. D'où, le développement d'une mythologie familiale et personnelle et le désir de parvenir à une consécration littéraire qui s'avoue difficile à atteindre. Desormeaux fait montre d'une connaissance encyclopédique de l'époque romantique et du dix-neuvième siècle en général. Il appelle en renfort pour étayer sa vision du parcours de Dumas quantité de critiques, d'auteurs, d'articles journalistiques, de mémoires, dans l'essentiel de première main. Pour ce qui est des travaux de Dumas lui-même, toutefois, il limite assez nettement ses explorations à ceux que nous avons mentionnés, non sans faire allusion, il est vrai, avec plus ou moins de détails, à d'autres livres, mais en montrant une préférence certaine pour les textes préfaciels ou autobiographiques (qui se confondent souvent), ainsi que pour les articles journalistiques dans lesquels Dumas parlait volontiers de lui-même. Les mille et un fantômes est un ouvrage relativement peu étudié, et c'est fort bien de le voir ici discuté de manière tout à fait pertinente. Les Mémoires ont fait l'objet de bien plus de 
réflexions, mais il est vrai que leur volume considérable garantit qu'il y aura encore pour bien longtemps des réflexions et des commentaires intéressants à en tirer. Ceci dit, dans cette étude les œuvres ne sont utilisées au bout du compte que comme des portes d'entrée, susceptibles de laisser pénétrer le lecteur dans l'esprit, dans l'âme, ou plus modernement dans la psychologie de l'écrivain. On y découvre et passe en revue ses doutes, ses incertitudes, ses sentiments plus ou moins refoulés d'infériorité ou d'illégitimité, les affres de l'absence du nom - tout cela compensé, semble-t-il, par l'écriture, à des degrés divers chez le père et chez le fils. L'ouvrage propose ainsi par conséquent un portrait hypothétique de la psychologie de Dumas - portrait certainement intrigant, convaincant sans doute par moments. Mais il ne dit en définitive que très peu sur l'œuvre, comme si celle-ci n'existait pas en elle-même et n'avait pas d'intérêt ni de validité autonomes en dehors de la fonction unique qu'on lui attribue ici, qui est de faire vivre dans l'éternité le nom de l'auteur - nom incertain dans son esprit que l'on dépeint obsédé de problèmes de descendance, d'hérédité, de racines et d'origine. Il est d'ailleurs tout particulièrement aisé de remarquer à quel point, parmi les sources secondaires utilisées, dominent indiscutablement les sources biographiques et anecdotiques, alors que la critique littéraire dumasienne, considérable cependant depuis au moins une vingtaine d'années, est pratiquement absente ou ne remplit qu'un rôle discret d'appoint. Charles Grivel, Sarah Mombert, bien d'autres encore sont mentionnés une fois, mais jamais réellement cités, ou sont tout simplement absents. On a souvent cependant l'impression, surtout en lisant les réflexions de l'auteur sur l'enchevêtrement de l'autobiographique et du romanesque chez Dumas, ainsi que sur les stratégies d'autoreprésentation qu'il a mises en pratique, de lire du déjà-lu ailleurs avant. Déjà-lu pour nous du moins, mais certainement pas cité dans le livre, dont l'auteur fait preuve cependant d'une érudition considérable. Ceci n'est nullement pour suggérer que le critique se serait rendu coupable du "crime", tout relatif d'ailleurs et bien difficile à déterminer, de "plagiat ", dont il parle à bien des reprises concernant Dumas. Loin de là. Simplement, la critique de l'œuvre dumasienne ne peut que perdre d'importance et se faire oublier lorsque le sujet du livre est l'auteur lui-même, avant ses œuvres.

4 Sainte-Beuve, le grand pourfendeur de la «littérature industrielle» que Dumas représentait probablement mieux que quiconque à son époque, expliquait l'œuvre par l'homme. Desormeaux semble vouloir expliquer l'homme par l'œuvre et montrer dans ce cas leur équivalence pratiquement parfaite. Le sérieux de la démarche et les qualités de l'écriture font que la lecture de ce livre intéressera sans le moindre doute tout dixneuviémiste et tout passionné de littérature populaire. D'autres regretteront probablement que les centaines de volumes produits par Dumas, qui méritent souvent une évaluation susceptible de mettre en lumière leurs qualités (ou leurs défauts) proprement littéraires, soient mis ici au deuxième plan derrière la silhouette considérable de leur auteur. 


\section{AUTHOR}

VITTORIO FRIGERIO

Dalhousie University 